\title{
ATITUDE DO CONSUMIDOR EM RELAÇÃO A CULTURAS PROBIÓTICAS E ALIMENTOS PROBIÓTICOS
}

\section{CONSUMER ATTITUDE TOWARD PROBIOTIC CULTURES AND PROBIOTIC PRODUCTS}

\author{
Tatiana Colombo Pimentel ${ }^{1}$ \\ ${ }^{1}$ Engenheira de Alimentos, Instituto Federal do Paraná - Campus Ivaiporã - Brasil \\ tatipimentel@ hotmail.com
}

\begin{abstract}
Resumo
O objetivo deste trabalho foi avaliar junto aos consumidores da região Norte do Paraná as crenças, atitudes e hábitos de consumo de culturas probióticas e produtos probióticos. Para isso aplicou-se um questionário a 100 pessoas, onde se verificou um baixo consumo deste tipo de produto. A avaliação de atitude revelou concordância que o consumo regular de produtos probióticos melhora o funcionamento do intestino, pode diminuir os níveis de colesterol, pode aumentar a absorção de cálcio proveniente da dieta e reforça os mecanismos de defesa do corpo. No entanto, os consumidores ainda acreditam que componentes funcionais, como os probióticos, têm capacidade de prevenir ou tratar doenças e não estão convencidos de que a sua ingestão não desencadeia reações adversas à saúde após o consumo. De uma forma geral, a atitude dos entrevistados foi positiva, porém, é recomendável reforçar a comunicação ao consumidor das alegações de propriedades funcionais e de saúde de culturas probióticas.
\end{abstract}

Palavras-chave: consumidor; probióticos; saúde.

\section{Introdução}

Nas últimas décadas houve um aumento na conscientização quanto à saúde e qualidade de vida, o que encorajou as pessoas a praticar exercícios físicos, adquirir hábitos de alimentação saudáveis e diminuir o consumo de alimentos ricos em açúcar, sal e gordura (PINHEIRO et al., 2005). Paralelamente a este fenômeno, observa-se um acelerado desenvolvimento de alimentos que promovem o bem-estar, melhoram a saúde e reduzem o risco de doenças (MUSSATO e MANCILHA, 2007).

Alimento funcional é um alimento semelhante em aparência ao alimento convencional; consumido como parte da dieta usual; capaz de produzir demonstrados efeitos metabólicos ou fisiológicos úteis na manutenção de uma boa saúde física e mental, podendo auxiliar na redução de riscos de doenças crônico-degenerativas, além das funções nutricionais básicas (HEALTH CANADA, 1998). 
O mercado de funcionais representa uma demanda nova do consumidor informado, que busca alimentos diferenciados (STRINGHETA et al., 2007). Mundialmente, foi estimado um crescimento no total de vendas deste tipo de alimento de 75 bilhões de dólares em 2007 para 109 bilhões de dólares em 2010 (GBA, 2007). De acordo com IFIC (2005) (International Food Information Council Foundation) os probióticos constituem exemplos de componentes funcionais.

O consumo de produtos adicionados de culturas probióticas está relacionado a efeitos benéficos em humanos, como: síntese de vitaminas e proteínas pré-digeridas; aumento na absorção de cálcio; inibição de patógenos no intestino; alívio da constipação; redução da intolerância à lactose; redução da incidência de tumores intestinais; diminuição do colesterol sérico; entre outros (SANDERS, 2003; SHEIL et al., 2007).

A atitude deriva da composição de componentes cognitivos, afetivos e conativos. Como componente cognitivo agrupa-se as crenças e conhecimento que a pessoa possui referente a um objeto. Já o componente afetivo congrega os sentimentos de uma pessoa sobre determinado objeto da atitude. Por fim, o elemento conativo é representado pelas tendências de comportamento da pessoa quanto ao objeto da atitude. Assim sendo, pode-se dizer que a atitude é algo distinto, porém, relacionada aos seus componentes (FERASSO, 2007).

Verificar a atitude do consumidor possibilita à empresa o desenvolvimento de embalagem, de rótulo e de alegações de campanhas publicitárias que informem sobre características e propriedades do alimento, de forma a elevar a expectativa do consumidor em relação ao produto e incentivar a compra do mesmo (BEHRENS e DA SILVA, 2004). O objetivo do presente trabalho foi avaliar junto aos consumidores de nível educacional médio e alto da região Norte do Paraná as crenças, atitudes e hábitos em relação a culturas probióticas e produtos probióticos.

\section{Material e métodos}

Por se tratar de pesquisa envolvendo seres humanos, a metodologia descrita a seguir cumpriu os termos da Resolução n. 196 (10/10/1996), do Conselho Nacional de Saúde (BRASIL, 1996).

\section{Levantamento de crenças, opiniões e nível de conhecimento de consumidores}

Crenças, opiniões e nível de conhecimento dos consumidores sobre culturas probióticas e produtos probióticos foram inicialmente levantados através de um questionário, segundo modelo sugerido por Mueller (1986) e utilizado por Behrens e Da Silva (2004). Este questionário solicitava que os indivíduos formulassem cinco frases que encerrassem crenças e conhecimentos sobre culturas probióticas, e foi aplicado a universitários das cidades de Maringá e Londrina, Estado do 
Paraná, durante o período de janeiro a março de 2010. Um total de 50 indivíduos (28 do sexo feminino e 22 do sexo masculino) respondeu o referido questionário.

\section{Elaboração da escala de avaliação de atitude}

A partir do conjunto de crenças, conhecimentos e opiniões obtido na etapa inicial da pesquisa (item 2.1), uma escala de avaliação de atitude em relação a culturas probióticas e produtos probióticos foi construída, de acordo com a metodologia proposta por Mueller (1986). Esta escala continha 26 itens (frases) sobre culturas probióticas e produtos probióticos, sendo metade deles de caráter negativo e a outra metade de caráter positivo (BEHRENS e DA SILVA, 2004).

A validade e poder de segmentação da escala formulada foram testados seguindo-se procedimento sugerido por Mueller (1986) e Hair (2005). Nesta etapa, a escala de atitude foi testada com um grupo de 50 indivíduos, consumidores dos produtos em questão, sendo 25 do sexo masculino e 25 do sexo feminino, alunos de graduação, pós-graduação e funcionários de universidades das cidades de Maringá e Londrina. Esses indivíduos foram abordados nos campus universitários e solicitados a responder à pesquisa, de acordo com sua disponibilidade, interesse, conhecimento e consumo de produtos probióticos. Os respondentes leram cada uma das frases e expressaram o grau com que concordaram ou discordaram de cada item através da escala de Likert de 7 pontos ( $1=$ discordo muito; $7=$ concordo muito) (BEHRENS e DA SILVA, 2004).

Após a coleta das respostas, os itens positivos da escala receberam os valores atribuídos a cada categoria: na categoria "concordo muito" foi computada a nota 7; na categoria "concordo moderadamente", foi computada nota 6 , e assim por diante até nota 1 na categoria "discordo muito". Os itens negativos foram inversamente computados: a categoria "concordo muito" recebeu nota 1 e a categoria “discordo muito" recebeu nota 7 (BEHRENS e DA SILVA, 2004).

Os dados assim computados foram estatisticamente analisados calculando-se o índice de discriminação de cada item; que consistiu no coeficiente de correlação de Pearson obtido ao correlacionarem-se as notas que os respondentes deram para cada item com a soma total das notas obtidas por todos os itens da escala, conforme descrito por Mueller (1986). Itens apresentando índices de discriminação próximos ou inferiores a zero deveriam ser eliminados do questionário.

\section{Avaliação final}

Através do procedimento de validação da escala descrito no item 2.2, os 26 itens originais sobre culturas probióticas e produtos probióticos foram mantidos, pois todos apresentaram índices de discriminação positivos e distantes de zero. 
Assim, um questionário final de pesquisa, contendo os 26 itens sobre culturas probióticas e produtos probióticos foi reaplicado a um total de 100 indivíduos, recrutados entre alunos de graduação e pós-graduação de universidades de Maringá e Londrina.

Com a finalidade de caracterizar a população entrevistada e obter informações sobre o hábito de consumo de produtos probióticos, foram incluídas no questionário perguntas sobre a faixa etária, sexo e grau de escolaridade, bem como o nível de consumo de produtos probióticos. A freqüência de consumo foi avaliada através de uma escala estruturada em 7 pontos (1= nunca experimentei; $7=$ consumo muito).

\section{Análise e quantificação dos dados}

Os dados de atitude obtidos na avaliação final foram estatisticamente analisados da seguinte forma (BEHRENS e DA SILVA, 2004):

- os itens positivos da escala receberam os valores atribuídos a cada categoria: na categoria "concordo muito" foi computada a nota 7; na categoria "concordo moderadamente", foi computada nota 6 , e assim por diante até nota 1 na categoria "discordo muito". Os itens negativos foram inversamente computados: a categoria "concordo muito" recebeu nota 1 e a categoria "discordo muito" recebeu nota 7. Com isso, foram determinados os índices de discriminação para cada item da escala de atitude;

- porcentagem de respostas de cada categoria da escala de Likert, para cada item do questionário;

- cálculo do coeficiente de Cronbach, também conhecido como "alfa":

Sendo:

$$
\text { Alfa }=(\mathrm{k} / \mathrm{k}-1)\left[1-\left(\sum \mathrm{Si}^{2} / \mathrm{S}_{\mathrm{t}}^{2}\right)\right]
$$

$$
\begin{aligned}
& \mathrm{k}=\text { número de itens que compõem a escala } \\
& \mathrm{S}_{\mathrm{i}}^{2}=\text { variância de cada item da escala } \\
& \mathrm{S}_{\mathrm{t}}^{2}=\text { variância total }
\end{aligned}
$$

\section{Resultados e discussão}

\section{Caracterização da população estudada}

Dos 100 indivíduos respondentes do questionário final, 64 pessoas foram do sexo feminino e 36 do sexo masculino, sendo $82 \%$ com idade entre 16 e 25 anos e $18 \%$ entre 26 e 45 anos. Quanto ao nível de escolaridade, $70 \%$ dos entrevistados possuíam o terceiro grau incompleto e 30\% pós- 
graduação. Assim, por se tratar de um segmento da população universitária, verificou-se que os indivíduos participantes da pesquisa foram basicamente jovens consumidores com nível de instrução razoável.

\section{Consumo de produtos probióticos}

A frequência de consumo de produtos probióticos pelos consumidores está apresentada na Figura 1.

Figura 1 - Frequência de consumo de produtos probióticos

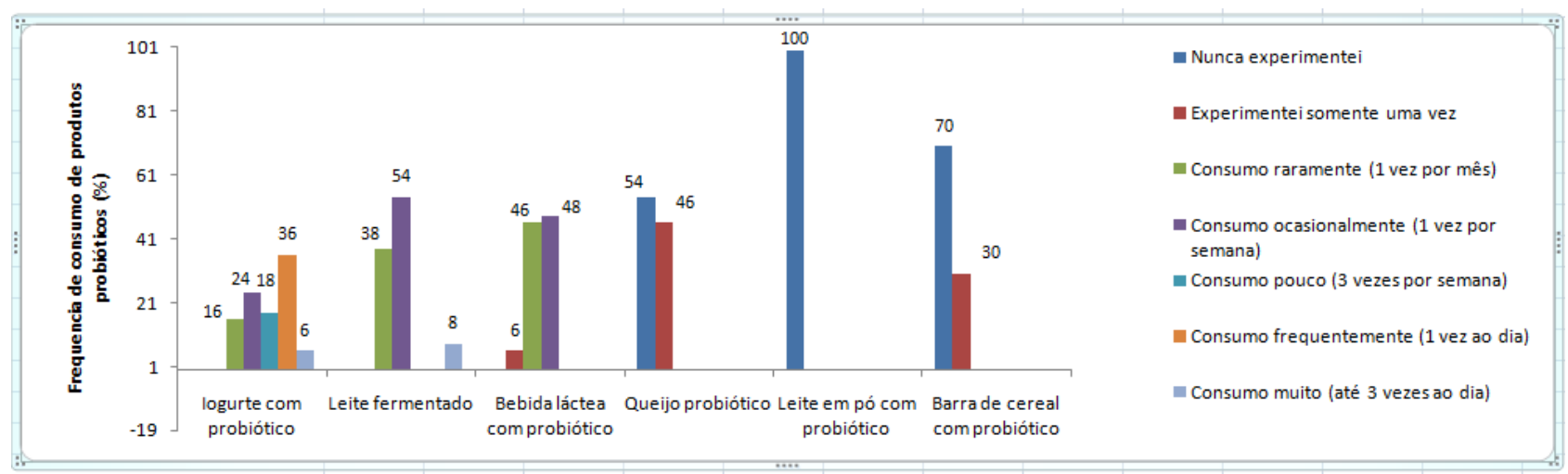

O produto mais consumido foi o iogurte com probiótico, sendo que $84 \%$ dos consumidores relataram consumir este tipo de produto mais do que 1 vez por semana, onde $42 \%$ deles o faziam no mínimo uma vez ao dia. O consumo de leite fermentado e bebida láctea com probiótico foi relatado como sendo de raro a ocasional por mais de $90 \%$ dos consumidores, ou seja, em torno de uma vez por semana a uma vez por mês.

Os produtos menos consumidos pelos entrevistados ou então nunca consumidos foram o queijo com probiótico, leite em pó com probiótico e barra de cereal com probiótico.

Pelos resultados obtidos é possível observar um baixo consumo de produtos probióticos pelos consumidores, muito aquém do estabelecido para exercer efeitos funcionais e de saúde. Para exercer um impacto benéfico à saúde, preconiza-se o consumo diário de 100 gramas de um produto adicionado de probiótico em quantidades superiores a $10^{6}$ UFC por $\mathrm{mL}$ ou $\mathrm{mg}$ (TOMA e POKROTNIEKS, 2006).

O baixo consumo relatado pode estar relacionado à faixa etária estudada (82\% com idade entre 16 e 25 anos). De acordo com Fisberg et al. (2000), o comportamento alimentar vincula-se fortemente aos padrões manifestados pelo grupo etário ao qual pertence. Omissão de refeições, consumo de alimentos com elevado conteúdo energético e pobre em nutrientes e ingestão precoce de bebidas alcoólicas são comuns na faixa etária estudada. 
Avaliação da atitude dos consumidores com relação às culturas probióticas e produtos probióticos

As Tabelas 1, 2 e 3 apresentam os itens que compuseram a escala de medida de atitude com relação às culturas probióticas e produtos probióticos, seus respectivos escores médios, frequência de respostas em cada categoria da escala de Likert e o índice de discriminação.

Como discutido no item 2.3, no teste preliminar do questionário, nenhum dos itens da escala de medida de atitude sobre culturas probióticas e produtos probióticos apresentou índice de discriminação negativo, e poucos se aproximaram de zero. Assim, foi decidido aplicar o questionário final com os 26 itens originalmente formulados.

Entretanto, quando os resultados dos 100 respondentes finais foram computados e analisados, seis itens $(4,15,19,21,23$ e 24) apresentaram resultados próximos de zero (Tabela 1). Itens com índices próximos a zero indicam que estes não contribuem para o poder da escala em segmentar os respondentes; provavelmente por não avaliarem questões relevantes ao tema da escala, ou por avaliarem crenças ou idéias de grau de concordância ou discordância praticamente consensual entre os indivíduos (BEHRENS e DA SILVA, 2004).

De fato, $100 \%$ dos entrevistados concordaram em um grau igual ou superior a "concordo" que "o consumo regular de probióticos melhora o funcionamento do intestino" (item 4). Além disso, a maioria dos entrevistados discordou das afirmações 19, 21 e 24: "não é necessária maior divulgação sobre os benefícios das culturas probióticas"(100\%); “a ingestão de probióticos aumenta a incidência de doenças" (96\%); e "mulheres grávidas ou em período de amamentação não podem consumir produtos probióticos" (82\%).

Os itens com índice de discriminação próximo a zero foram, então, eliminados da escala final, pois diminuíam o poder de segmentação e a confiabilidade da escala, sendo esta última avaliada através do coeficiente de Cronbach (BEHRENS e DA SILVA, 2004).

Tabela 1 - Proporção de respostas (\%) e escore médio dos ITENS RETIRADOS da escala de atitude em relação a culturas probióticas e produtos probióticos

\begin{tabular}{|c|c|c|c|c|c|c|c|c|c|}
\hline \multicolumn{10}{|c|}{ Proporção de respostas $(\%)^{*}$} \\
\hline Itens & $1(\%)$ & $2(\%)$ & $3(\%)$ & $4(\%)$ & $5(\%)$ & $6(\%)$ & $7(\%)$ & $\begin{array}{l}\text { Escore Médio e } \\
\text { Desvio Padrão }\end{array}$ & $\begin{array}{c}\text { Índice de } \\
\text { Discriminação }\end{array}$ \\
\hline 4. & 0 & 0 & 0 & 0 & 44 & 2 & 54 & $6,10 \pm 0,99$ & 0,0001 \\
\hline 15. & 30 & 10 & 0 & 28 & 22 & 0 & 10 & $3,42 \pm 1,98$ & 0,0001 \\
\hline 19. & 82 & 2 & 16 & 0 & 0 & 0 & 0 & $1,34 \pm 0,74$ & 0,0001 \\
\hline 21. & 80 & 6 & 10 & 4 & 0 & 0 & 0 & $1,38 \pm 0,83$ & 0,0001 \\
\hline 23. & 0 & 0 & 26 & 36 & 28 & 0 & 10 & $4,32 \pm 1,16$ & 0,0001 \\
\hline 24. & 30 & 0 & 52 & 18 & 0 & 0 & 0 & $2,58 \pm 1,10$ & 0,0001 \\
\hline
\end{tabular}

*1 - Discordo muito; 2- Discordo moderadamente; 3- Discordo; 4- Não concordo nem discordo; 5- Concordo; 6- Concordo moderadamente; 7- Concordo muito. 4. O consumo regular de probióticos melhora o funcionamento do intestino; 15. Todos os iogurtes são iguais, independentemente dos microrganismos presentes; 19 . Não é necessária maior divulgação sobre os benefícios das culturas probióticas; 21 . A ingestão de probióticos aumenta a incidência de doenças; 23. A ingestão de iogurtes com probióticos não pode ser utilizada em substituição a medicamentos em caso de constipação intestinal; 24. Mulheres grávidas ou em período de amamentação não podem consumir produtos probióticos 
A escala de atitude em relação a culturas probióticas e produtos probióticos, considerando-se os 20 itens restantes relacionados nas Tabelas 2 e 3, apresentou coeficiente de Cronbach igual a 0,98. De acordo com Hair (2005), uma escala que apresente este coeficiente igual ou superior a 0,60 é um instrumento confiável para se medir atitudes sobre o objeto relacionado. Assim, a escala apresentada nas Tabelas 2 e 3 pode ser considerada confiável e de poder discriminativo satisfatório uma vez que todos os 20 itens da escala obtiveram índices de discriminação positivos (BEHRENS e DA SILVA, 2004).

Tabela 2 - Proporção de respostas (\%) e escore médio dos ITENS POSITIVOS da escala de atitude em relação a culturas probióticas e produtos probióticos

\begin{tabular}{|c|c|c|c|c|c|c|c|c|c|}
\hline \multicolumn{10}{|c|}{ Proporção de respostas $(\%)^{*}$} \\
\hline Itens & $\begin{array}{c}1 \\
(\%)\end{array}$ & $\begin{array}{c}2 \\
(\%)\end{array}$ & $\begin{array}{c}3 \\
(\%)\end{array}$ & $\begin{array}{c}4 \\
(\%)\end{array}$ & $\begin{array}{c}5 \\
(\%)\end{array}$ & $\begin{array}{c}6 \\
(\%)\end{array}$ & $\begin{array}{c}7 \\
(\%)\end{array}$ & $\begin{array}{l}\text { Escore Médio e } \\
\text { Desvio Padrão }\end{array}$ & $\begin{array}{c}\text { Índice de } \\
\text { Discriminação }\end{array}$ \\
\hline $\begin{array}{l}\text { 1. Uma dieta equilibrada, incluindo } \\
\text { produtos probióticos, é fundamental } \\
\text { para o bom funcionamento do } \\
\text { organismo humano }\end{array}$ & 0 & 0 & 0 & 0 & 0 & 10 & 90 & $6,90 \pm 0,30$ & 0,36 \\
\hline $\begin{array}{l}\text { 2. Os probióticos exercem um impacto } \\
\text { benéfico à saúde dos consumidores } \\
\text { quando a sua concentração nos produtos } \\
\text { apresenta níveis adequados }\end{array}$ & 2 & 0 & 10 & 10 & 44 & 8 & 26 & $5,22 \pm 1,38$ & 0,64 \\
\hline $\begin{array}{l}\text { 3. Os probióticos podem ser } \\
\text { encontrados naturalmente no trato } \\
\text { intestinal de indivíduos saudáveis }\end{array}$ & 0 & 0 & 0 & 0 & 16 & 0 & 84 & $6,68 \pm 0,74$ & 0,54 \\
\hline $\begin{array}{l}\text { 5. O consumo de probióticos pode } \\
\text { diminuir os níveis de colesterol }\end{array}$ & 0 & 0 & 0 & 6 & 46 & 18 & 30 & $5,72 \pm 0,96$ & 0,46 \\
\hline $\begin{array}{l}\text { 6. O consumo de probióticos pode } \\
\text { aumentar a absorção de cálcio } \\
\text { proveniente da dieta }\end{array}$ & 0 & 0 & 0 & 6 & 52 & 14 & 28 & $5,64 \pm 0,96$ & 0,46 \\
\hline $\begin{array}{l}\text { 7. O consumo de probióticos pode } \\
\text { prevenir e tratar doenças }\end{array}$ & 4 & 0 & 0 & 10 & 34 & 0 & 52 & $5,78 \pm 1,49$ & 0,62 \\
\hline $\begin{array}{l}\text { 8. As culturas probóticas estimulam a } \\
\text { multiplicação de bactérias benéficas, } \\
\text { reforçando os mecanismos de defesa do } \\
\text { corpo }\end{array}$ & 4 & 0 & 6 & 6 & 44 & 10 & 30 & $5,36 \pm 1,46$ & 0,60 \\
\hline $\begin{array}{l}\text { 9. Os rótulos que trazem a expressão } \\
\text { "Lactobacilos vivos" chamam a atenção } \\
\text { porque sei que são alimentos mais } \\
\text { saudáveis }\end{array}$ & 0 & 0 & 0 & 10 & 30 & 28 & 32 & $5,82 \pm 1,00$ & 0,36 \\
\hline $\begin{array}{l}\text { 10. A ingestão de probióticos é a } \\
\text { solução para a constipação intestinal }\end{array}$ & 0 & 6 & 0 & 0 & 24 & 10 & 60 & $6,12 \pm 1,34$ & 0,46 \\
\hline $\begin{array}{l}\text { 11. O consumo de probióticos pode } \\
\text { melhorar a intolerância à lactose }\end{array}$ & 10 & 0 & 28 & 4 & 34 & 0 & 24 & $4,48 \pm 1,87$ & 0,36 \\
\hline $\begin{array}{l}\text { 12. O consumo de probióticos deve } \\
\text { estar associado a uma alimentação } \\
\text { saudável e à prática de exercícios } \\
\text { físicos }\end{array}$ & 0 & 0 & 6 & 14 & 42 & 0 & 38 & $5,50 \pm 1,29$ & 0,56 \\
\hline $\begin{array}{l}\text { 13. Quanto maior o consumo de } \\
\text { produtos probióticos melhor será o } \\
\text { efeito benéfico encontrado }\end{array}$ & 0 & 0 & 6 & 0 & 66 & 14 & 14 & $5,30 \pm 0,93$ & 0,46 \\
\hline
\end{tabular}

*1 - Discordo muito; 2- Discordo moderadamente; 3- Discordo; 4- Não concordo nem discordo; 5- Concordo; 6- Concordo moderadamente; 7Concordo muito. 
Tabela 3 - Proporção de respostas (\%) e escore médio dos ITENS NEGATIVOS da escala de atitude em relação a culturas probióticas e produtos probióticos

\begin{tabular}{|c|c|c|c|c|c|c|c|c|c|}
\hline \multicolumn{10}{|c|}{ Proporção de respostas $(\%)^{*}$} \\
\hline Itens & $\begin{array}{c}1 \\
(\%) \\
\end{array}$ & $\begin{array}{c}2 \\
(\%) \\
\end{array}$ & $\begin{array}{c}3 \\
(\%) \\
\end{array}$ & $\begin{array}{c}4 \\
(\%) \\
\end{array}$ & $\begin{array}{c}5 \\
(\%) \\
\end{array}$ & $\begin{array}{c}6 \\
(\%) \\
\end{array}$ & $\begin{array}{c}7 \\
(\%) \\
\end{array}$ & $\begin{array}{l}\text { Escore Médio e } \\
\text { Desvio Padrão }\end{array}$ & $\begin{array}{c}\text { Índice de } \\
\text { Discriminação }\end{array}$ \\
\hline $\begin{array}{l}\text { 14. As culturas probióticas podem } \\
\text { irritar ou agredir a mucosa intestinal }\end{array}$ & 4 & 0 & 20 & 38 & 28 & 0 & 10 & $4,26 \pm 1,30$ & 0,56 \\
\hline $\begin{array}{l}\text { 16. A ingestão de probióticos não } \\
\text { necessita ser diária }\end{array}$ & 22 & 0 & 44 & 24 & 10 & 0 & 0 & $3,00 \pm 1,24$ & 0,54 \\
\hline $\begin{array}{l}\text { 17. Crianças não devem consumir } \\
\text { produtos adicionados de probióticos }\end{array}$ & 24 & 10 & 24 & 32 & 10 & 0 & 0 & $2,94 \pm 1,34$ & 0,56 \\
\hline $\begin{array}{l}\text { 18. Não é preciso desenvolver mais } \\
\text { alimentos adicionados de probióticos } \\
\text { para compra }\end{array}$ & 18 & 10 & 72 & 0 & 0 & 0 & 0 & $2,54 \pm 0,78$ & 0,39 \\
\hline $\begin{array}{l}\text { 20. O sabor de alimentos adicionados } \\
\text { de probióticos é diferente do produto } \\
\text { sem adição }\end{array}$ & 12 & 0 & 48 & 0 & 24 & 10 & 6 & $3,78 \pm 1,65$ & 0,50 \\
\hline $\begin{array}{l}\text { 22. Alimentos adicionados de } \\
\text { probióticos apresentam seu rótulo } \\
\text { dificultado e de difícil entendimento }\end{array}$ & 10 & 18 & 10 & 0 & 40 & 6 & 16 & $4,24 \pm 1,94$ & 0,54 \\
\hline $\begin{array}{l}25 . \quad \text { Os alimentos probióticos } \\
\text { geralmente apresentam um custo mais } \\
\text { elevado que os demais }\end{array}$ & 0 & 0 & 0 & 18 & 48 & 10 & 24 & $5,40 \pm 1,04$ & 0,39 \\
\hline $\begin{array}{l}\text { 26. A ingestão de probióticos para a } \\
\text { melhoria do trânsito intestinal faz } \\
\text { com que o intestino fique dependente } \\
\text { deste produto para funcionar } \\
\text { normalmente }\end{array}$ & 4 & 14 & 24 & 0 & 24 & 24 & 10 & $4,38 \pm 1,77$ & 0,49 \\
\hline
\end{tabular}

Os entrevistados concordaram em um grau igual ou superior a "concordo moderadamente" $(100 \%)$ com o item 1 e concordaram em um grau igual ou superior a "concordo" (80\%) com o item 12; ou seja, que "uma dieta bem equilibrada, incluindo produtos probióticos, é fundamental para o bom funcionamento do organismo humano" e que "o consumo de probióticos deve estar associado a uma alimentação saudável e à prática de exercícios físicos". Os resultados indicam que os consumidores estão cientes de que a mudança dos hábitos alimentares, com a ingestão de refeições rápidas e altamente calóricas, aliada ao estilo de vida sedentário de muitos indivíduos, contribui para o aumento da incidência de doenças (SAAD et al., 2011).

Em relação ao conhecimento sobre culturas probióticas, verificou-se um nível satisfatório; já que $78 \%$ dos entrevistados concordaram que "os probióticos exercem um impacto benéfico à saúde dos consumidores quando a sua concentração nos produtos apresenta níveis adequados" (item 2) e $100 \%$ que "os probióticos podem ser encontrados naturalmente no trato intestinal de indivíduos saudáveis" (item 3).

Quanto aos benefícios à saúde associados às culturas probióticas, a maioria dos entrevistados concordou com os itens 5, 6 e 8, ou seja, estão de acordo que "o consumo de probióticos pode diminuir os níveis de colesterol" (94\%); "aumentar a absorção de cálcio proveniente da dieta" (94\%) e "estimular a multiplicação de bactérias benéficas, reforçando os 
mecanismos de defesa do corpo" (84\%). Estes resultados indicam que estes indivíduos encontramse bastante cientes da associação entre consumo de alimentos probióticos e benefícios à saúde. De fato, estudos indicam que a suplementação da dieta com probióticos pode assegurar o equilíbrio microbiano intestinal e, consequentemente, a manutenção da saúde do hospedeiro, ou mesmo reduzir o risco do aparecimento de determinadas doenças, como por exemplo, câncer, doenças cardiovasculares, osteoporose, entre outras (SAAD et al., 2011).

No entanto, os consumidores não tinham certeza se "o consumo de probióticos pode melhorar a intolerância à lactose" (item 11), já que 58\% concordaram e 38\% discordaram da afirmação, a qual era verdadeira. Má absorção de lactose é uma condição na qual o principal carboidrato do leite não é completamente hidrolisado aos seus monossacarídeos, glicose e galactose, devido à ausência da enzima $\beta$-D-galactosidade no intestino de algumas pessoas. Bactérias probióticas, produzem esta enzima, que hidrolisa a lactose, resultando em um aumento da tolerância por produtos lácteos (SHAH, 2007). Infelizmente esta informação parece não ter ainda atingido a população entrevistada, apesar desta ser considerada de alto nível educacional para os padrões da média nacional. Esses resultados apontam a necessidade de maior veiculação das citadas informações na mídia nacional (BEHRENS e DA SILVA, 2004).

Observa-se para o item 14 alta frequiência de entrevistados (38\%) que expressaram suas opiniões através da categoria "não concordo/nem discordo" da escala de Likert. Isto indica que um grande número de indivíduos pareceu desconhecer ou não estar certo da afirmação proposta por este item que "as culturas probióticas podem irritar ou agredir a mucosa intestinal". Além disso, 58\% dos entrevistados concordaram que "a ingestão de probióticos para melhoria do trânsito intestinal faz com que o intestino fique dependente deste produto para funcionar normalmente" (item 26). As afirmações contidas nos itens 14 e 26 eram falsas, já que os probióticos são microrganismos encontrados naturalmente no trato intestinal de indivíduos saudáveis, portanto, não irritam ou agridem a mucosa intestinal e não tornam o indivíduo dependente de seu consumo para que o intestino funcione regularmente. Estudos clínicos controlados não revelaram efeitos maléficos causados por esses microorganismos (SAAD et al., 2011). Esses resultados indicam que os consumidores não estão convencidos de que a ingestão de culturas probióticas é segura e que esses microorganismos não apresentam efeitos adversos à saúde após o consumo.

Com relação aos itens 7 e 10 houve concordância por $86 \%$ e 94\% dos entrevistados, respectivamente, ou seja, os consumidores acreditam que "o consumo de probióticos pode prevenir e tratar doenças" (item 7) e que "a ingestão de probióticos é a solução para a constipação intestinal" (item 10). A legislação brasileira não permite alegações de saúde que façam referência à cura ou prevenção de doenças, pois as doenças crônico-degenerativas são multifatoriais e o controle de apenas um componente como a dieta não é realmente suficiente para a prevenção (BRASIL, 1999; 
LAJOLO, 2005). Portanto, deve-se pensar que os componentes funcionais presentes nos alimentos teriam capacidade de reduzir o risco de aparecimento de algumas doenças e não preveni-las ou tratá-las.

Os resultados indicam que os consumidores ainda crêem que componentes funcionais, como o probióticos, teriam a capacidade de prevenção ou tratamento de doenças crônico-degenerativas, o que é um fato preocupante. Segundo Pinto (2008), há evidências de tendência das empresas de provocar a confusão do consumidor em relação à natureza do produto, associando-o a medicamento, aumentando assim o risco sanitário, pois o consumidor deixa de buscar ou retarda a procura de tratamento adequado para suas necessidades.

Verifica-se que 94\% dos consumidores concordaram que "quanto maior o consumo de probióticos melhor será o efeito benéfico encontrado" (item 13), sendo que esta é uma afirmativa falsa. Segundo Gallo e Puglia (2008), o efeito dos probióticos não é cumulativo, ou seja, não aumenta com um maior consumo de alimentos probióticos. No entanto, probióticos ingeridos em quantidades elevadas normalmente não oferecem risco à saúde (ANTUNES, 2009), o que faz com que a crença dos consumidores nesta afirmação falsa não seja tão preocupante.

Os entrevistados estavam bem informados com relação ao público alvo que poderia consumir o produto; pois 58\% discordaram que "crianças não devem consumir produtos adicionados de probióticos" (item 17). Segundo Antunes (2009), o leite materno contém naturalmente bactérias potencialmente probióticas (bifidobactérias e lactobacilos). Portanto, parece não haver restrição para consumo de probióticos desde a infância, podendo os mesmos ser adicionados em fórmulas lácteas. Em geral, todas as pessoas podem consumir produtos probióticos, com especial recomendação para idosos, que precisam de uma reposição de bactérias benéficas, perdidas ao longo da vida.

A importância da ingestão diária de probióticos também é de conhecimento dos entrevistados, pois 66\% destes discordaram do item 16, o qual afirmava que "a ingestão de probióticos não precisa ser diária”. Porém, percebe-se na frequência de consumo (item 3.2), um baixo consumo de produtos probióticos, fato que pode ser atribuído ao custo e a escassa variedade destes produtos disponíveis no mercado, se restringindo quase que exclusivamente a produtos lácteos fermentados. De fato, $82 \%$ dos entrevistados concordaram que "os alimentos probióticos geralmente apresentam um custo mais elevado que os demais" (item 25) e 100\% discordaram que "não é preciso desenvolver mais alimentos adicionados de probióticos disponíveis para compra" (item 18).

Em relação à palatabilidade dos produtos probióticos, $60 \%$ dos entrevistados discordaram do item “o sabor de alimentos adicionados de probióticos é diferente do produto sem adição" (item 20). Portanto, a maioria dos consumidores considera os produtos probióticos como tendo sabor similar 
aos produtos convencionais, o que é importante para a comercialização de alimentos funcionais, já que estudos mostram que o sabor é o primeiro direcionador para a escolha de um alimento, seguido pela consideração em relação à saúde. Esses mesmos estudos indicaram também que os consumidores não possuem interesse em consumir um produto funcional se os ingredientes adicionados puderem conferir sabores desagradáveis, mesmo que sejam adicionadas vantagens em relação à saúde (CRUZ et al., 2011).

Em relação à rotulagem, a escala de atitude apresentava dois itens. Verificou-se que $62 \%$ dos entrevistados concordaram que "os alimentos adicionados de probióticos apresentam seu rótulo dificultado e de difícil entendimento" (item 22); e 90\% que "os rótulos que trazem a expressão Lactobacilos vivos chamam a atenção, pois se sabe que são alimentos mais saudáveis" (item 9). Os resultados indicam que há uma falha no desenvolvimento de produtos adicionados de probióticos, pois os rótulos deveriam ser esclarecedores aos consumidores em geral; e que as alegações apresentadas nos rótulos, quando claras, contribuem com as vendas dos produtos enriquecidos com nutrientes benéficos à saúde, como os probióticos.

\section{Conclusões}

Os resultados da presente pesquisa indicaram que o consumo de produtos probióticos foi baixo entre os consumidores que responderam ao questionário. Poucos indivíduos reportaram o consumo freqüente deste tipo de produto, sendo que o iogurte com probióticos, leite fermentado e bebida láctea com probiótico foram indicados como os produtos mais conhecidos e consumidos pelos mesmos. Por outro lado, queijo com probiótico, leite em pó com probiótico e barra de cereal com probiótico foram produtos praticamente desconhecidos desses indivíduos.

$\mathrm{Na}$ avaliação da atitude, os entrevistados concordaram que o consumo regular de probióticos melhora o funcionamento do intestino, pode diminuir os níveis de colesterol, pode aumentar a absorção de cálcio proveniente da dieta e reforça os mecanismos de defesa do corpo. No entanto, ainda há extrapolação nas propagandas com relação às alegações de propriedades funcionais, induzindo os consumidores a acreditarem que componentes funcionais, como os probióticos, têm capacidade de prevenir ou tratar doenças. Além disso, os consumidores não estão convencidos de que os probióticos não poderiam causar efeito adverso após o consumo.

Em conclusão, as indústrias alimentícias que desejam lançar no mercado produtos probióticos devem investir tanto nos aspectos sensoriais do produto, como na divulgação dos benefícios do consumo de culturas probióticas à saúde humana, a fim de levantar expectativas positivas nos consumidores e estimular a compra e o consumo destes produtos. 


\begin{abstract}
The aim of this study was to evaluate beliefs, attitudes and habits of consumption of probiotic cultures and probiotic products by consumers in the Northern region of Paraná. For this, a questionnaire was applied to 100 people, where it was verified that there was a low consumption of such products. The attitude assessment showed agreement that regular consumption of probiotic products improves the functioning of the intestine, may lower cholesterol levels, may increase the absorption of calcium from the diet and strengthens the body's defense mechanisms. However, consumers still believe that functional components, such as probiotics, have the capacity to prevent or treat diseases and are not convinced that the intake does not trigger adverse health reactions after consumption. In general, the attitude of respondents was positive, however, it is recommended to strengthen communication to consumer of health and functional properties of probiotic cultures.
\end{abstract}

Key-words: consumer; probiotic; beliefs.

\title{
Referências
}

ANTUNES, A. E. C. Prebióticos e probióticos: cuide do seu intestino. 2009. Disponível em: <http://idmed.uol.com.br/alimentacao-saudavel/prebioticos-e-probioticos-cuide-do-seu-intestino.html>. Acesso em set. 2011.

BEHRENS, J. H.; DA SILVA, M. A. A. P. Atitude do consumidor em relação a soja e produtos derivados. Revista Ciência e Tecnologia de Alimentos, v. 24, n. 3, p. 431-439, 2004. http://dx.doi.org/10.1590/S010120612004000300023

BRASIL. Ministério da Saúde. Conselho Nacional de Saúde. Resolução n. 196, de 10 de outubro de 1996. Diário Oficial da União, Brasília, 10 out.1996. 9p.

BRASIL. Ministério da Saúde, Agência Nacional de Vigilância Sanitária (ANVISA). Resolução n. 18 de 30 de Abril de 1999. Disponível em: <http://e-legis.bvs.br/leisref/public/showAct.php?id109>. Acesso em set. 2011.

CRUZ, A. G.; CADENA, R. S.; FARIA, J. A. F.; BOLINI, H. M. A. Análise sensorial de produtos alimentícios probióticos e prebióticos. In: SAAD, S. M. I; CRUZ, A. G.; FARIA, J. A. F. Probióticos e prebióticos em alimentos: fundamentos e aplicações tecnológicas. São Paulo: Livraria Varela, 2011. p. 195-222.

FERASSO, M. Influenciando as atitudes dos consumidores. 2007. Disponível em: <www.administradores.com.br/informe-se/artigos/influenciando-as-atitudes-dos-consumidores/20118/>. Acesso em set. 2011.

FISBERG, M. Hábitos alimentares na adolescência. 2000. Disponível em: < http://br.monografias.com/trabalhos2/habitos-alimentares-adolescencia/habitos-alimentares-adolescencia.shtml>. Acesso em set. 2011.

GAllo, V. C.; PUGLIA, L. C. Probióticos, fibra alimentar e prebióticos. 2008. Disponível em: <http://nutrociencia.com.br/textos_mostra.asp?vid=1083ecid=4>. Acesso em set. 2011.

GBA. Functional foods and drinks market to reach U.S. \$109 billion by 2010. Global Industry Analystis, San Jose, Calif., 2007.

HAIR, J. F.; ANDERSON, R. E.; TATHAM, R. L.; BLACK, W. C. Análise multivariada de dados. 5 ed. (trad.). Porto Alegre: Bookman, 2005. 
HEALTH CANADA. 1998. Policy paper: nutraceuticals/ functional foods and health claim on foods. Otawa, Ontario, Canada. Health Canada, Health Protection Branch, Terapeutic Products Programme and Food Directorate. Disponível em: <http:// www.hc-sc.gc.ca〉. Acesso em set. 2011.

INTERNATIONAL FOOD INFORMATION COUNCIL FOUNDATION. IFIC. Alimentos funcionales. 2005. Disponível em: <http://www.ific.org/sp/nutrition/functional/index.cfm?renderforprint=1>. Acesso em set. 2011.

LAJOLO, F. M. Alimentos funcionais. Uma visão geral. In: ANGELIS, R.C. A importância dos alimentos vegetais na proteção da saúde: fisiologia da nutrição protetora e preventiva de enfermidade degenerativa. 2 ed., São Paulo: Atheneu, 2005. p. 175-181.

MUELLER, D. J. Measuring social attitudes: a handbook for researchers and practioners. New York: Teachers College, 1986. 179p.

MUSSATTO, S. I; MANCILHA, I. M. Non-digestible oligosaccharides: a review. Carbohydrate Polymers, v. 68, n. 3, p. 587-597, 2007. http://dx.doi.org/10.1016/j.carbpol.2006.12.011

PINHEIRO, M. V. S; OLIVEIRA, M. N; PENNA, A. L. B; TAMIME, A. Y. The effect of different sweeteners in lowcalorie yogurts - a review. International Journal of Dairy Technology, v. 58, n. 4, p. 193-199, 2005. http://dx.doi.org/10.1111/j.1471-0307.2005.00228.x

PINTO, M. A. O. Aspectos legais e análise de conteúdo de propagandas impressas de alimentos com alegações de propriedades funcionais. Viçosa, 2008. 327p. Tese (Doutorado em Ciência e Tecnologia de Alimentos) - Universidade Federal de Viçosa.

SAAD, S. M. I.; BEDANI, R.; MAMIZUKA, E. M. Benefícios à saúde dos probióticos e prebióticos. In: SAAD, S. M. I.; CRUZ, A. G.; FARIA, J. A. F. Probióticos e prebióticos em alimentos: fundamentos e aplicações tecnológicas. São Paulo: Livraria Varela, 2011.

SANDERS, M. E. Probiotics: considerations for human health. Nutrition Reviews, v. 61, n. 3, p. 91-99, 2003. http://dx.doi.org/10.1301/nr.2003.marr.91-99

SHAH, N. P. Functional cultures and health benefits. International Dairy Journal, v. 17, n. 11, p. 1262-1277, 2007. http://dx.doi.org/10.1016/j.idairyj.2007.01.014

SHEIL, B.; SHANAHAN, F.; O`MAHONY, L. Probiotic effects on inflammatory bowel disease. Journal of Nutrition, v. 137, n. 3, p. 819-824, 2007.

STRINGHETA, P. C; VILELA, M. A. P; OLIVEIRA, T. T.; NAGEM, T. J. Alimentos "Funcionais": conceitos, contextualização e regulamentação. Juiz de Fora: Templo, 2007. 246p.

TOMA, M. M.; POKROTNIEKS, J. Probiotics as functional food: microbiological and medical aspects. Acta Universitatis Latvienses, v. 710, p. 117-129, 2006.

Submetido em 08 set 2011, Aceito para publicação em 16 ago. 2012. 